\title{
Landing Stability Simulation of a 1/6 Lunar Module with Aluminum Honeycomb Dampers
}

\author{
Van Lai Pham*, Jun Zhao* and Nam Seo Goo** \\ Department of Advanced Technology Fusion, Division of Interdisciplinary studies, Konkuk University, Seoul, 143-701, Korea \\ Jae Hyuk Lim*** and Do-Soon Hwang***
}

Korea Aerospace Research Institute, Daejeon, 305-333, Korea

\section{Jung Sun Park**}

Aerospace and Mechanical Engineering, Korea Aerospace University, Gyeonggi-do, 412-791, Korea

\begin{abstract}
The Korea Aerospace Research Institute plans to launch a lunar module by 2025, and so is carrying out a preliminary study. Landing stability on the lunar surface is a key design factor of a lunar module. In this paper, a 1/6 scale model of a lunar module is investigated, for its landing stability on non-level surfaces. The lunar module has four tripod legs, with aluminum honeycomb shock absorbers in each leg strut. ADAMS ${ }^{\mathrm{TM}}$, the most widely used multi-body dynamics and motion analysis software, is used to simulate the module's lunar landing. Three types of dampers in the struts (rigid, viscous, and aluminum honeycomb dampers), and two types of lunar surfaces (rigid and elastic) are considered. The Sforce function is adopted, to model the aluminum honeycomb dampers. Details on the modeling and analysis of the landing stability of the 1/6 scale lunar module and the simulation results are provided in this paper.
\end{abstract}

Key words: Lunar module, ADAMS, Landing stability, Aluminum honeycomb damper

\section{Introduction}

With scientific and technological advancements, many countries or organizations are carrying out extensive research on space and the Moon. The Moon, Earth's natural satellite, has re-emerged as the focus of space research recently, because of its abundant natural resources, and high potential as a future territory. The Korea Aerospace Research Institute (KARI) is planning to launch a lunar exploration module by 2025 , and has already proceeded with a preliminary study on a potential lunar module.

In the overall design of a lunar lander, the analysis of the touchdown dynamics during the landing stage is an important task, and the design of a landing system that can absorb the high landing impact energy is indispensable, for guaranteeing the stability and safety of the lunar module, and to protect the devices inside it. In the 1950s, mission flights in the Solar System were started. The use of a parachute or a balloon was considered, to reduce the landing velocity and impact between the lunar module and the lunar surface. But neither of these methods was effective. An aluminum honeycomb damper was not used until the late 1960s. The honeycomb damper was researched, to satisfy the strict requirements of spacecraft design. Apollo 11 first successfully landed on the lunar surface in July, 1969 [1]. Since the 1980s, the aluminum honeycomb shock absorber has been widely used in the aerospace industry.

Analysis study of the touchdown dynamic modeling and simulation of a lunar lander vehicle received great interest, and its touchdown stability was investigated $[2,3]$. In this
This is an Open Access article distributed under the terms of the Creative Commons Attribution Non-Commercial License (http://creativecommons.org/licenses/by$\mathrm{nc} / 3.0 /$ which permits unrestricted non-commercial use, distribution, and reproduction in any medium, provided the original work is properly cited.

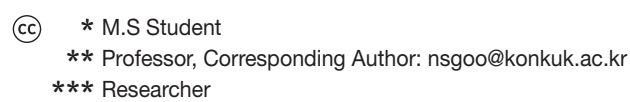


paper, the $1 / 6$ model of a lunar module with the aluminum honeycomb shock absorber, and the lunar surface, are both constructed in $\mathrm{ADAMS}^{\mathrm{TM}}$. The modeling method of the aluminum honeycomb damper and the lunar surface has been investigated. The landing stability of the lunar module on level and non-level surfaces is calculated. From the simulations, the proper method for modeling the damper and the lunar surface is investigated, and the landing stability of a $1 / 6$ lunar module is presented.

\section{Problem statement}

\subsection{Preliminary design of a lunar module}

KARI made a preliminary design of a lunar module that is to land on the Moon. Its total mass was $250 \mathrm{~kg}$. For testing, the $1 / 6$ lunar module was considered in terms of mass, because the Moon's gravity is one-sixth that of Earth. Fig. 1 shows the 1/6 lunar module, which consists of a truss structure and a dummy mass. The truss structure has four tri-pod legs. Each leg has one primary strut and two secondary struts to absorb shock energy. A dummy mass represents payloads, such as the electronic system, fuel, and guidance system.

This study investigates the landing stability of the $1 / 6$ lunar module under the Earth's gravity. First, the simple equation for an overturn was considered. Second, ADAMS ${ }^{\mathrm{TM}}$ was used to solve the multi-body dynamics of the landing of the 1/6 lunar module. A CAD model constructed in CATIA was imported into ADAMS ${ }^{\mathrm{TM}}$, for use in simulations.

\subsection{Theoretical landing stability}

Fig. 2 shows the calculation of the tip-over angle of the $1 / 6$ lunar module. Table 1 provides the definition of the symbols used in Fig. 2. Equilibrium equations can be derived for the configuration shown in Fig. 2.

From the moment equilibrium at leg \#1:

$Z_{2} \cdot a+G \cdot \sin \alpha \cdot h_{g}-G \cdot \cos \alpha \cdot \frac{a}{2}=0$

The module loses stability, when the reaction force $\mathrm{Z}_{2}=0$, so Eq. 1 becomes:

$G \cdot \sin \alpha \cdot h_{g}-G \cdot \cos \alpha \cdot \frac{a}{2}=0$

Then, the condition of module stability can be derived:

$G \cdot \sin \alpha \cdot h_{g}-G \cdot \frac{a}{2} \cdot \cos \alpha \leq 0 \Leftrightarrow \tan \alpha \leq \frac{a}{2 \cdot h_{g}}$

Also, the glide condition of the module can be derived:

$G \cdot \sin \alpha \cdot \leq P_{f}=G \cdot f \cdot \cos \alpha \Leftrightarrow \tan \alpha \leq f$

By considering the parameters $a=1486(\mathrm{~mm})$ and $h_{g}=$ $557(\mathrm{~mm})$ of the $1 / 6$ lunar module defined in Fig. 1, the safe

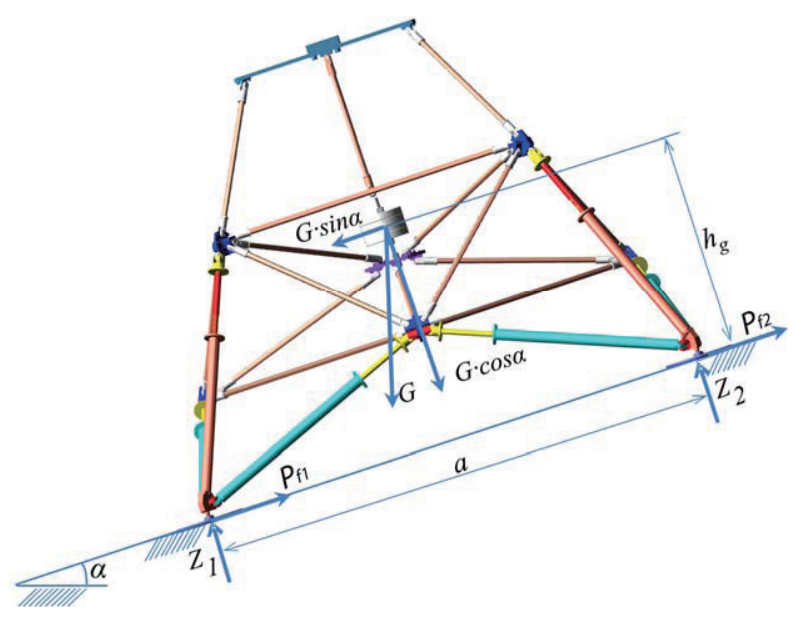

Fig. 2. Tip-over angle of the lunar module

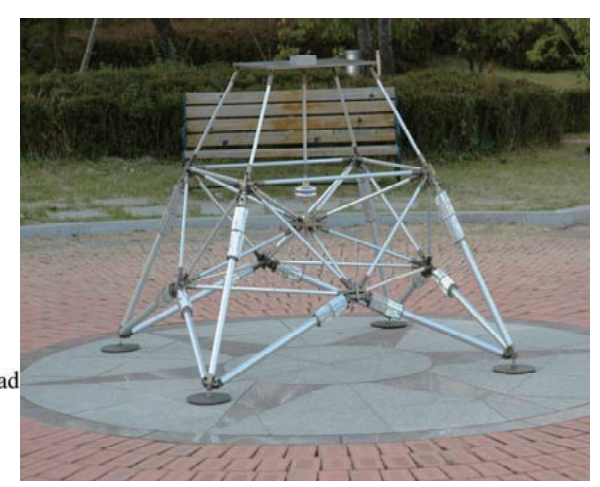

(b) Real photograph

Fig. 1. 1/6 lunar module with truss structure, and dummy mass. 
condition can be calculated: the angle of the tilt surface should be smaller than $40.3^{0}$

\subsection{Aluminum honeycomb shock absorber}

In the design of a lunar module, the landing gear system is considered to be one of the most important components. The landing gear must absorb the impact energy as much as possible, to protect the payloads, when the lunar module touches down on the lunar surface. Historically, several shock absorbers were invented, tested, and used for lunar modules: Ranger 4 in 1962 used a lunar capsule covered with a balsawood impact-limiter; Ranger 9 in 1965 consisted of a hexagonal aluminum frame, where the propulsion and power units were mounted; and Luna 8 in 1965 used an airbag [4]. However, for landing missions that carry human(s) to the Moon, energy absorbers should be designed to achieve a soft landing. The landing module needs to decelerate to a reasonable velocity, before hitting the Moon. While the balloon mechanism that was used for the landing on Mars is interesting and thought provoking, a parachute cannot be used for landing on the Moon, because the Moon's atmosphere is very thin. Therefore, to overcome this limitation, an aluminum honeycomb shock absorber was considered a good solution. Apollo 11 , the first spacecraft that brought men to land on the Moon, and returned them to Earth successfully [1], used an aluminum honeycomb shock absorber. Consequently, an aluminum honeycomb shock absorber was adopted for the present $1 / 6$ lunar module. Fig. 3 shows the details of the landing gear, which is comprised of one primary strut, two secondary struts supporting the primary strut, and one footpad.

In addition to the analysis of the landing stability of the 1/6 lunar module, an analysis of the aluminum honeycomb shock absorber was carried out, for its characterization. Fig. 4 shows the crush test results of a developed aluminum honeycomb shock absorber. They revealed that $80 \%$ of the total length was used for energy absorption, and that the compression force was constant, after the initial stage of compression.

The shock absorber has two honeycombs of different crush strengths: the honeycomb of the first stage has small crush strength, and that of the second stage has large crush strength. The main reason for using the two-honeycomb design is that it can reduce the peak acceleration, as well as absorb the whole impact energy. The honeycomb with the small crush strength can absorb the impact energy at the moment of touchdown, reduce the peak acceleration, and protect important payloads. Then, the honeycomb with the high crush strength can absorb the whole impact energy. Transition from the first stage to the second stage places artificial shock on the lunar module. To avoid this, the ratio of the crush strengths between the first and second stages should be minimized.

Fig. 5 shows the assumed crush strength (force) versus displacement relation, for use in simulations. The assumed crush force of the aluminum honeycomb shock absorbers was roughly approximated by doing several simulation landings of a 10 degree tilted lunar module for two landing

Table 1. Definition of parameters and dimensions defined in Fig. 2

\begin{tabular}{ccc}
\hline Symbol & Explanation & Value \\
\hline $\mathrm{G}$ & Gravity force of the module & $40(\mathrm{~kg})$ \\
$\mathrm{Z}_{1}$ & Reaction force at leg \#1 & \\
$\mathrm{Z}_{2}$ & Reaction force at leg \#2 & \\
$\mathrm{a}$ & $\begin{array}{l}\text { Distance between 2 legs } \\
\text { from lunar surface to the central of }\end{array}$ & $1486(\mathrm{~mm})$ \\
$\mathrm{h}_{\mathrm{g}}$ & $\begin{array}{c}\text { Dravity of the module } \\
\mathrm{P}_{\mathrm{f}}\end{array}$ & Friction force between legs and lunar surface \\
$\mathrm{f}$ & Static friction coefficient & \\
& & 0.85 \\
\hline
\end{tabular}

Table 2. Different types of aluminum honeycomb damper

\begin{tabular}{ccccc}
\hline Group & 1 & 2 & $\mathbf{3}$ & 4 \\
\hline $\begin{array}{c}\text { Crush force of first step } \\
(\mathrm{N})\end{array}$ & 200 & 200 & $\mathbf{3 0 0}$ & 300 \\
$\begin{array}{c}\text { Crush force of second } \\
\text { step (N) }\end{array}$ & 400 & 600 & $\mathbf{6 0 0}$ & 900 \\
$\begin{array}{c}\text { Length of first step } \\
(\mathrm{mm})\end{array}$ & 80 & 80 & $\mathbf{8 0}$ & 80 \\
$\begin{array}{c}\text { Length of second step } \\
(\mathrm{mm})\end{array}$ & 80 & 80 & $\mathbf{8 0}$ & 80 \\
\hline
\end{tabular}


modes (1-2-1 and 2-2, explained later), at different crush forces, as given in Table 2. The main condition is that the two aluminum honeycombs absorb the entire landing energy.

The simulation results revealed that the lunar module can land safely on the lunar surface, with the specification of shock absorbers in group 3 listed in Table 2 . In the primary struts, an aluminum honeycomb shock absorber consists of two compression stages during the energy shock absorption period: the first stage with soft crush strength of $300 \mathrm{~N}$ and length of $80 \mathrm{~mm}$, and the second one with hard crush strength of $600 \mathrm{~N}$ and length of $80 \mathrm{~mm}$. The area of the aluminum absorber in the preliminary design was $70 \times 68 \mathrm{~mm}^{2}$.

\subsection{Lunar surface}

The surface properties of the Moon affect the design of a lunar module; in particular, the design of the landing gear. Topographical and soil-properties of the Moon are available in the references on the lunar surface $[5,6]$. Fig. 6 shows the slope profile, protuberance, and modulus of the lunar surface. The topographical features consist of a mean surface slope of $6^{0}$ or less, and an effective slope of $12^{\circ}$ or less, including the effects of depressions or protuberances, and footpad penetration. The statistical description used most extensively was based on the topographical data from the Lunar Orbiter photograph of the most severe Apollo landing site.

Based on Apollo's experiment data, the modulus of subgrade reaction of the lunar surface was investigated, by using 776 analyses of boot prints (40\% analyses from Apollo 16). In the analyses, the area of applied load was $12.5 \mathrm{~cm}$ $\mathrm{x} 3 \mathrm{~cm}$, and the applied load per unit area was $q=7 \mathrm{kPa}$. If we call the boot print depth $d$, the modulus of subgrade reaction will be $k=q / d$. As shown in Fig. 6, the modulus of subgrade reaction of about $40 \%$ was $10 \mathrm{kPa} / \mathrm{cm}$. From this value and area of applied load, the stiffness of lunar surface was inferred as $k=41250 \mathrm{~N} / \mathrm{m}$. In the contact problem, the damping coefficient generally takes a value between $0.1 \%$ $1 \%$ of the stiffness coefficient. In this simulation, this value was selected as $412.5 \mathrm{~N} . \mathrm{s} / \mathrm{m}$, which is equal to $1 \%$ of the stiffness coefficient.

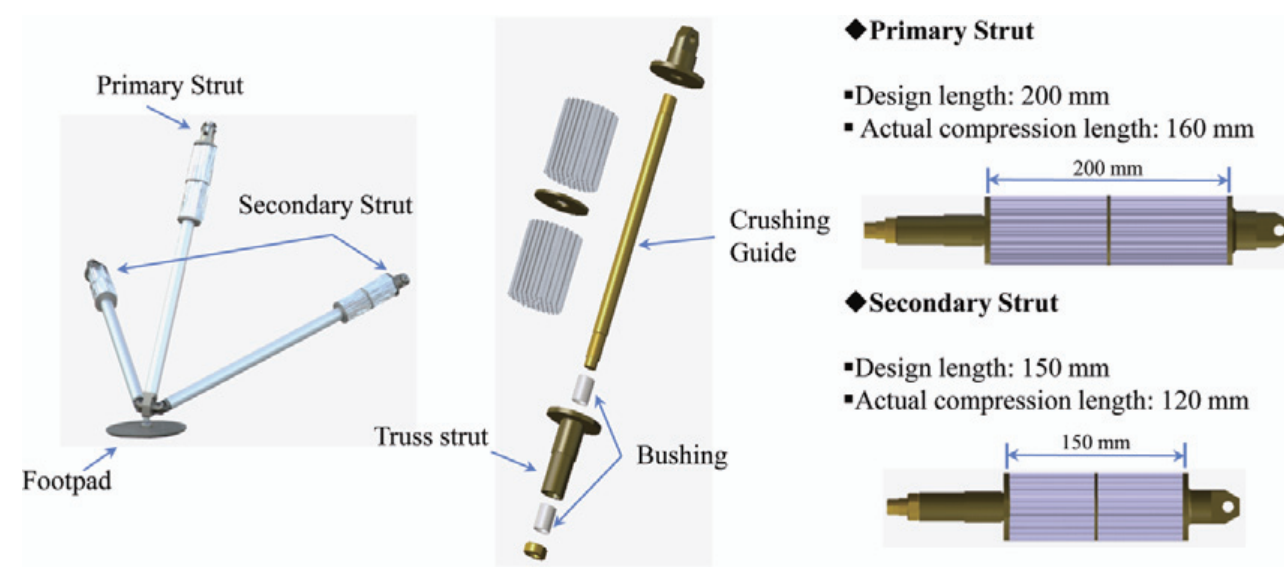

Fig. 3. Configuration of landing gear: primary strut, secondary struts, and footpad

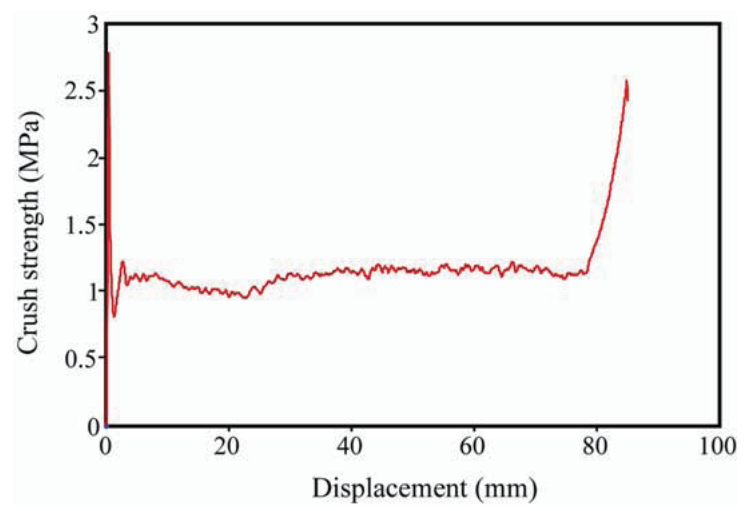

Fig. 4. Crush test result: crush force versus displacement relation

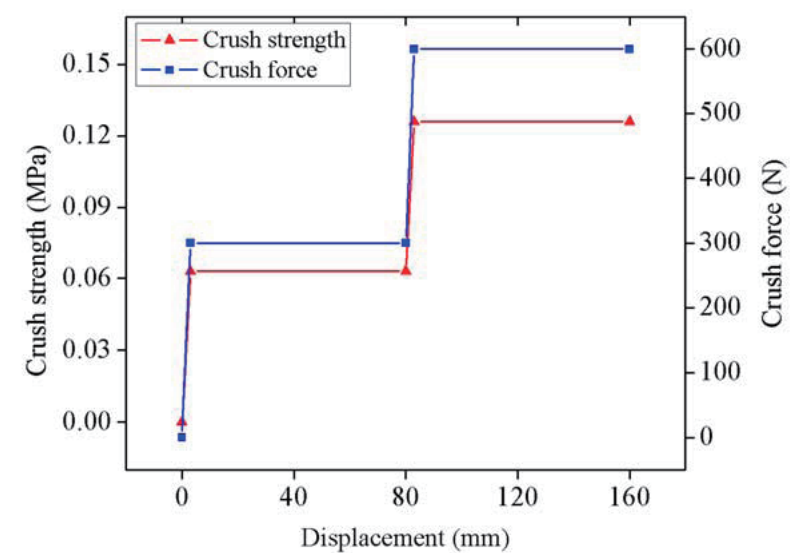

Fig. 5. Crush force-compression length curve of primary struts 


\section{Modeling}

\subsection{Lunar surface}

In this research, two types of lunar surfaces were considered in lunar surface simulations: the rigid surface, and the elastic surface. Fig. 7 shows the two types of lunar surfaces, along with the ADAMS model of the 1/6 lunar module. Even though a rigid surface does not reflect the actual property of the lunar surface, it can however serve as a reference. An elastic surface is two rigid surfaces connected by four springs.

Table 3 lists the specifications of the 1/6 lunar module, and the properties of the lunar surface. Based on the conditions, the spring constant of the elastic surface was

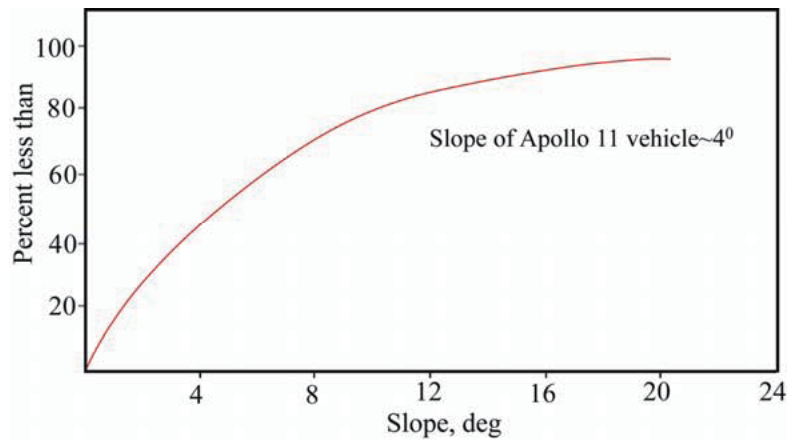

(a) Slope profile
$41250 / 4=10312.5 \mathrm{~N} / \mathrm{m}$. The lunar module constructed in ADAMS $^{\mathrm{TM}}$ was composed of nearly 720 rigid bodies, and many constraints, such as translational joints, spherical joints, fixed joints, and revolute joints, were placed on them.

\subsection{Strut modeling}

Three kinds of damper models were considered, to investigate the behavior of the struts: a rigid damper, a viscous damper, and an aluminum honeycomb damper. The rigid damper means that the strut does not have any damper, which was considered as a reference solution, like the rigid lunar surface. The viscous damper is a simple damper, which has a spring and a viscous damper, whose properties are listed in Table 3. To implement the aluminum honeycomb

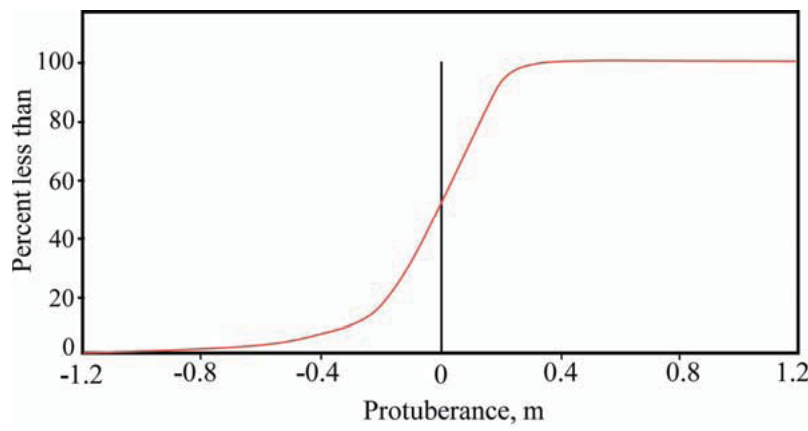

(b) Protuberance profile

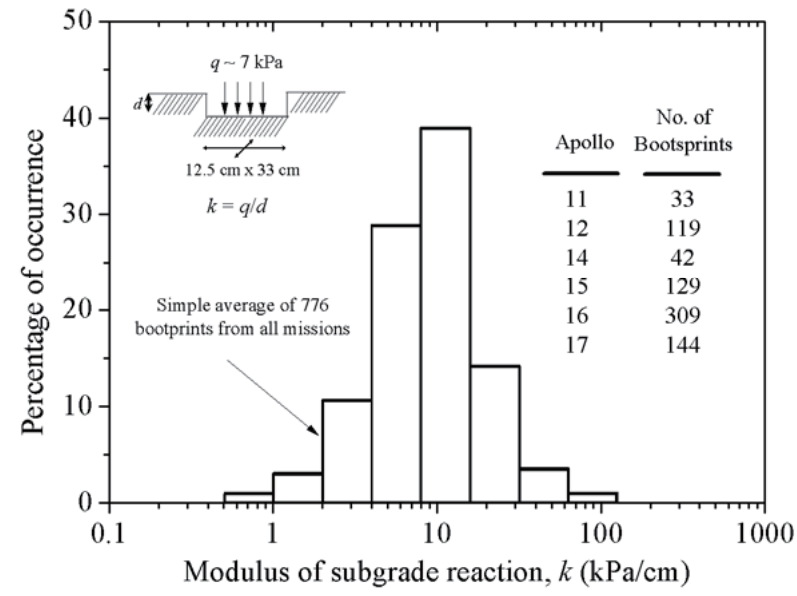

(c) Modulus of subgrade reaction

Fig. 6. Properties of lunar surface (Reproduction from $[5,6]$ )

Table 3. Specification of $1 / 6$ lunar module, and properties of lunar surface

\begin{tabular}{|c|c|c|c|c|c|c|}
\hline \multicolumn{3}{|c|}{ 1/6 lunar module } & \multicolumn{4}{|c|}{ Properties of Lunar surface } \\
\hline $\begin{array}{c}\text { Weight } \\
(\mathrm{kg})\end{array}$ & $\begin{array}{c}\text { Vertical impact } \\
\text { velocity } \\
(\mathrm{m} / \mathrm{s})\end{array}$ & $\begin{array}{c}\text { Gravity } \\
\left(\mathrm{m} / \mathrm{s}^{2}\right)\end{array}$ & $\begin{array}{l}\text { Dynamic } \\
\text { friction } \\
\text { coefficient }\end{array}$ & $\begin{array}{l}\text { Static } \\
\text { friction } \\
\text { coefficient }\end{array}$ & $\begin{array}{l}\text { Stiffness } \\
\text { coefficient } \\
(\mathrm{N} / \mathrm{m})\end{array}$ & $\begin{array}{c}\text { Damping } \\
\text { coefficient } \\
\text { (N.s/m) }\end{array}$ \\
\hline 42 & 4 & 9.8 & 0.4 & 0.85 & 41250 & 412.5 \\
\hline
\end{tabular}


damper, an algorithm was invented, using Sforce and Step functions. Fig. 8 shows the flow chart of the algorithm, and Table 4 lists one ADAMS example.

Basically, a landing gear consists of two main parts: the lower part, which impacts with the surface, and the upper part, which is able to absorb shock, to protect equipment. The upper and lower parts of a landing gear strut were considered in the algorithm as a cylinder and piston system. Hence, the compression process of the energy absorber is analogous to the movement between a piston and cylinder.

The compression process of an aluminum honeycomb damper consists of two stages: in the first stage, the shock absorber is compressed by a force of $300 \mathrm{~N}$. After the first stage, the shock absorber is subjected to a greater force of $600 \mathrm{~N}$ in the second stage. The compression lengths of the first and second stages are $80 \mathrm{~mm}$ in the primary struts. In the secondary struts, the compression lengths of the first and second stages are $60 \mathrm{~mm}$. A stable condition is reached, when the cylinder and piston move at the same velocity, which means no crush force is acting inside the shock absorber. In this study, the convergence velocity $\left(v_{\text {conv }}\right)$ for the stable condition is $5(\mathrm{~mm} / \mathrm{s})$.

\subsection{Simulation parameters}

When the landing simulation using ADAMS is performed, several parameters should be determined, for convergence and accuracy of solution. The impact between the lunar module and the lunar surface was modeled by the IMPACT function in ADAMS. The general form of the IMPACT force

Table 4. An example of aluminum honeycomb damper modeling with the flow chart in Fig. 8

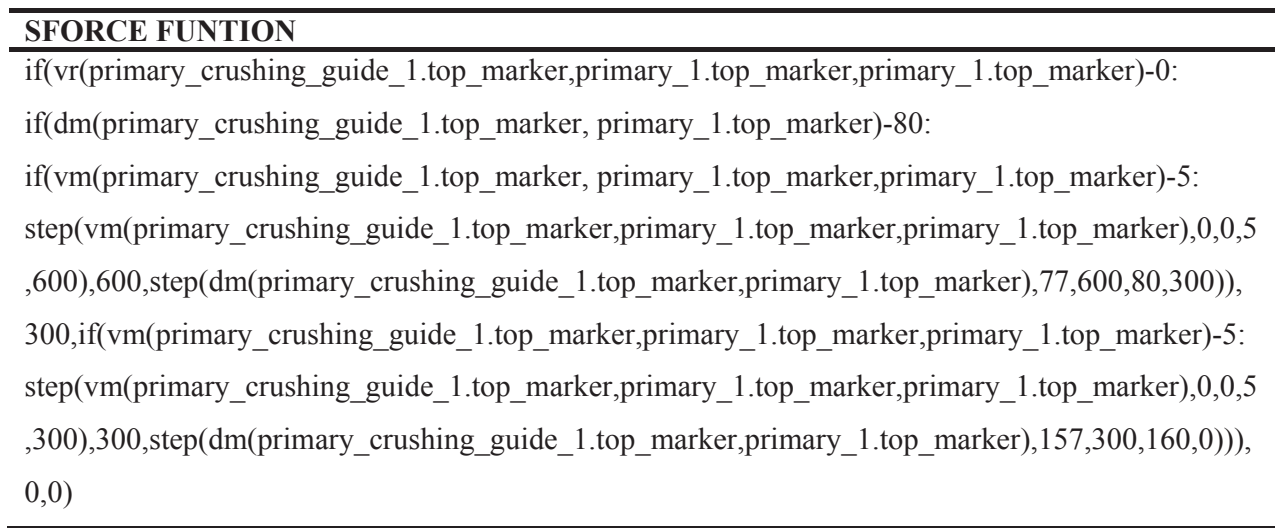

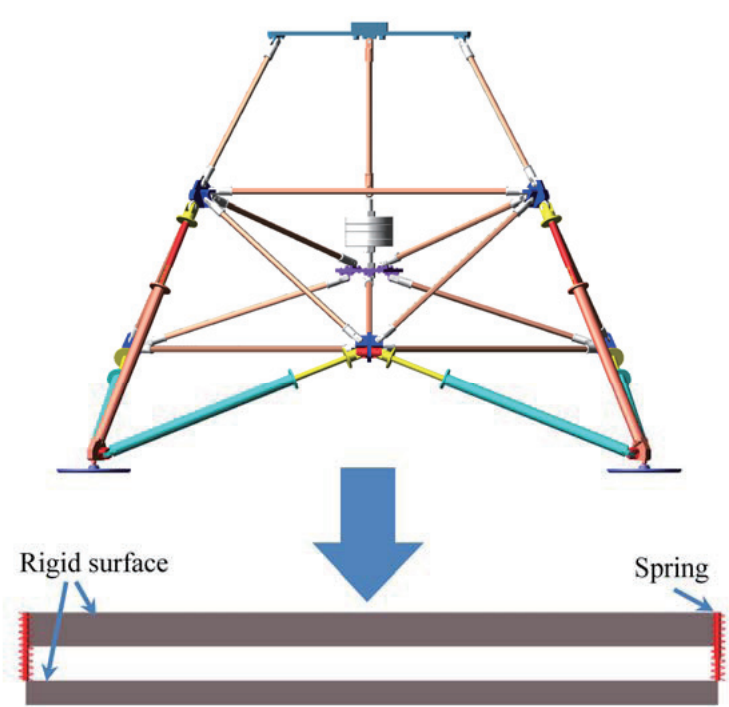

Elastic surface

Fig. 7. Lunar surface, and ADAMS model of $1 / 6$ lunar module

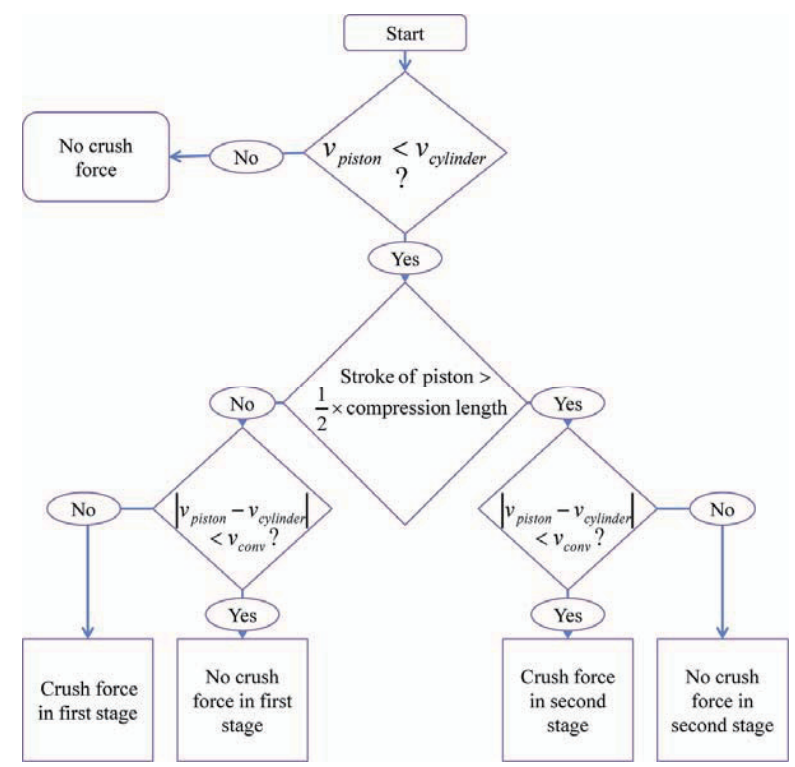

Fig. 8. Flow chart of modeling of an aluminum honeycomb damper in ADAMS 
function is calculated by:

$$
F=k g^{e}+\operatorname{Step}\left(g, 0,0, d_{\max }, c_{\max }\right) \frac{d g}{d t}
$$

where,

$g$ is the penetration of one geometry into another.

$\frac{d g}{d t}$ is the penetration velocity at the contact point.

$e$ is a positive real value denoting the force exponent.

$d_{\max }$ is a positive real value specifying the boundary penetration, used to apply the maximum damping coefficient $c_{\max }$.

The selection of parameters, such as time step, contact, penetration, and friction, is explained, as follows.

(1) Time step size

In simulation problems, it is important to select the proper time step size, in order to produce the correct results. However, for a model with hundreds of bodies all undergoing dynamic contact, we need to balance the convergence efficiency, against the required level of accuracy. The "best" value is determined by the trial and error method. In this work, the range of step size was chosen from 0.0005 to 0.01 second.

\section{(2) Contact condition}

The landing of a lunar module can be regarded as a dynamic contact problem between the lunar module and lunar surface. ADAMS ${ }^{\mathrm{TM}}$ simulates contacts between two bodies in several ways: solid-to-solid, curve-to-curve, pointto-curve, point-to-plane, curve-to-plane, sphere-to-plane, and sphere-to-sphere. In this study, a solid-to-solid contact was used.

Basically, contact stiffness depends on the shapes and properties of the two contact surfaces [7]. The static contact stiffness $\mathrm{K}$ between a rigid sphere and a deformable plate is calculated as [8]:

$$
K=\frac{d F}{d w}=\left(6 F R E^{2}\right)^{\frac{1}{3}}
$$

where, $\mathrm{F}$ is the impact force acting on the rigid sphere. $w=\left(9 \mathrm{~F}^{2} / 16 \mathrm{RE}^{2}\right)^{1 / 3}$, is indentation or contact area, will be circular. $E$ is the equivalent Young's Modulus: $1 / E=\left(1-v_{1}^{2}\right) /$
$E_{1}+\left(1-v_{2}^{2}\right) / E_{2} . E_{1}, v_{1}, R_{1}$ are Young's Modulus, Poisson's ratio, and the radius of part $1 . E_{2}, v_{2}, R_{2}$ are Young's Modulus, Poisson's ratio, and the radius of deformable plate of part 2, respectively. Actually, determining the correct contact stiffness for a complex shape is a difficult problem, which was not overemphasized in this study. From calculations for simple shapes, the contact stiffness can be estimated, and then used to examine the convergence behavior and accuracy. This method was performed several times, until an acceptable value was selected.

\section{(3) Penetration depth}

This value defines the penetration depth, for the case of full damping. ADAMS/Solver uses a cubic STEP function to increase the damping coefficient from zero, at zero penetration, to the coefficient of full damping, when the penetration reaches the damping penetration.

\section{(4) Force exponent}

The exponent of the force deformation is specified by a positive real value. For a stiffening spring characteristic, e $>1.0$; for a softening spring characteristic, $0<\mathrm{e}<1.0$. The exponent should normally be set to 1.5 or higher. In this simulation, this variable was assumed to range from 2 to 2.2

\section{(5) Friction}

In an impact problem, it is necessary to include the friction coefficient between two objects. In this study, this parameter was selected based on the information of the lunar surface. The dynamic and static friction coefficients of the lunar surface are 0.4 and 0.85 , in turn $[4,6]$.

\section{Results and discussions}

First, we compared the acceleration and velocity results of the body's center for several simulation cases listed in Table 5, to evaluate what kind of simulation model would be proper for use in a landing simulation, in terms of accuracy and calculation time. Second, 2-2 landing and 1-2-1 landing modes were investigated, for the landing of the lunar module at an inclined attitude on the surface. Third, the tip-over angle was investigated, to evaluate the stability of the $1 / 6$ lunar module.

Table 5. Simulation case

\begin{tabular}{cc}
\hline Types of lunar surface & Types of damper model \\
\hline Rigid surface & Rigid damper \\
Elastic surface & Viscous damper \\
& Al honeycomb damper \\
\hline
\end{tabular}


Van Lai Pham Landing Stability Simulation of a 1/6 Lunar Module with Aluminum Honeycomb Dampers

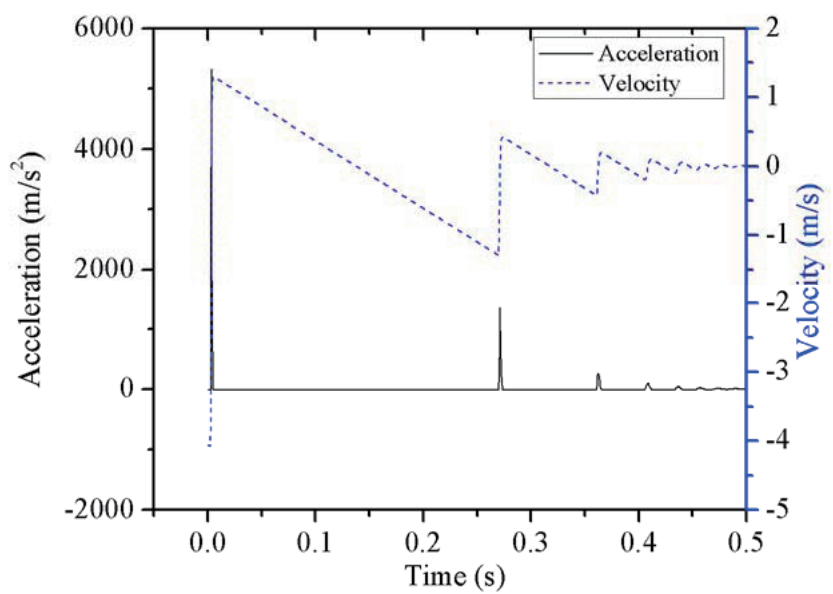

(a) Rigid lunar surface, and rigid damper

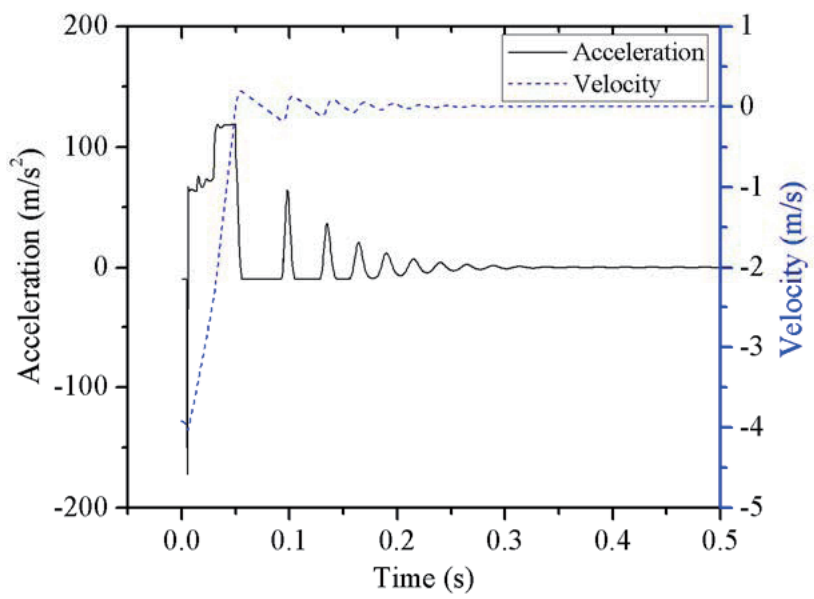

(c) Rigid lunar surface, and Al honeycomb damper

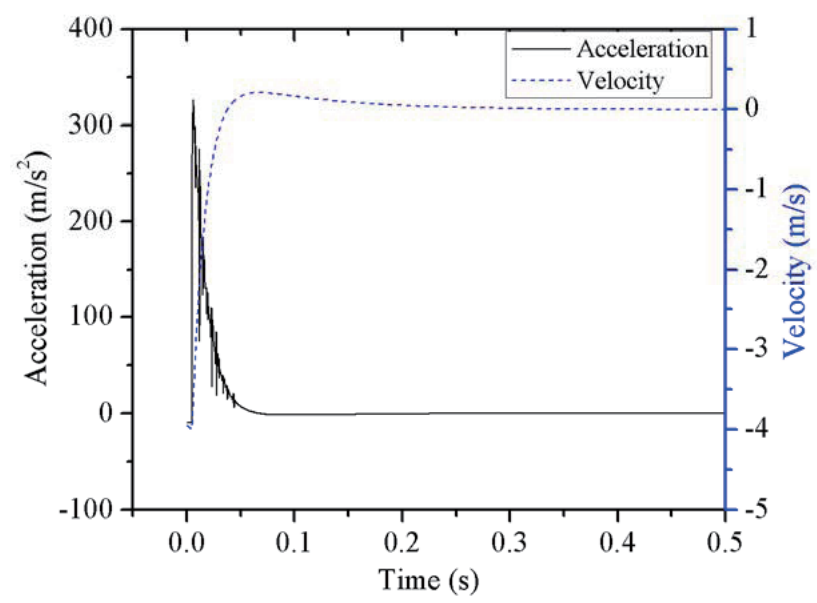

(e) Elastic lunar surface, and viscous damper

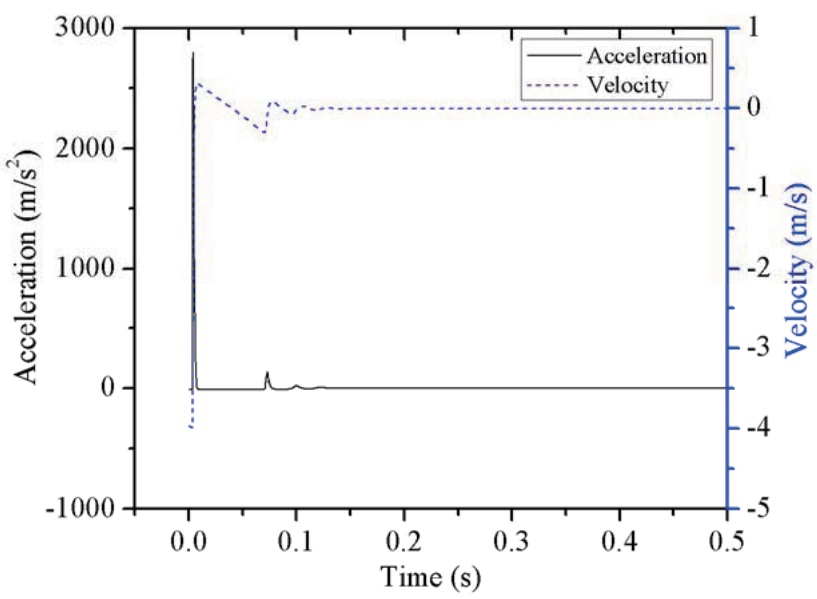

(b) Rigid lunar surface, and viscous damper

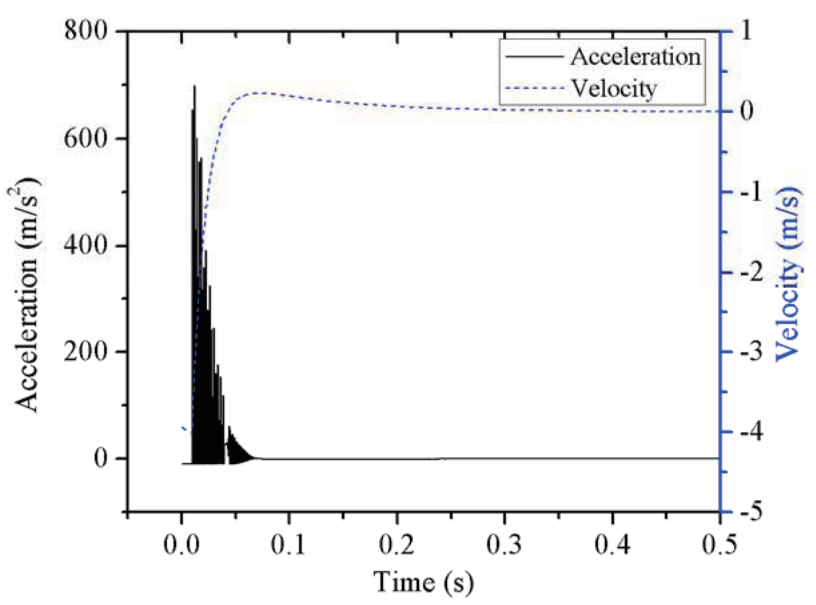

(d) Elastic lunar surface, and rigid damper

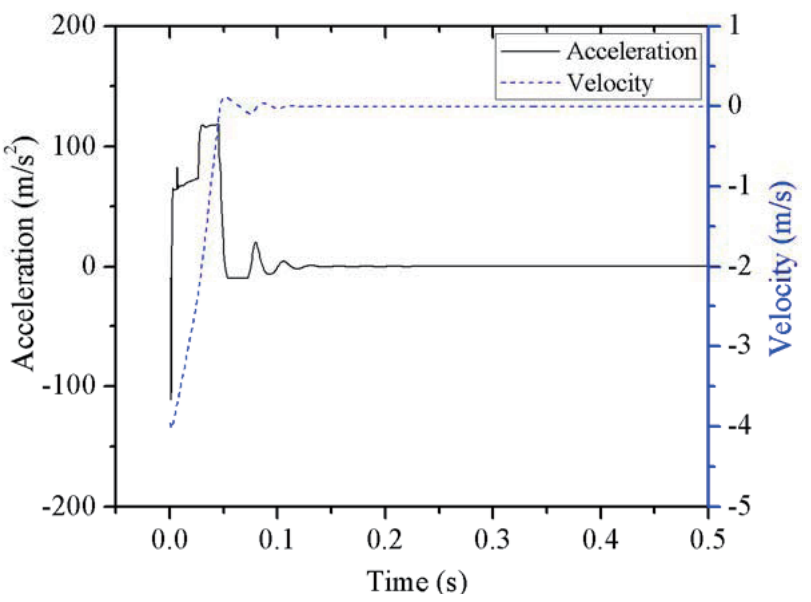

(f) Elastic lunar surface, and Al honeycomb damper

Fig. 9. Comparison of several analysis models for the lunar surface and damper 
In this study, a one-sixth size model (42 kg) under the Earth's gravity was considered to simulate the landing stability of a lunar module $(250 \mathrm{~kg})$ on the Moon. The multibody dynamics software, ADAMS, was adopted for the simulation, under the analysis condition listed in Table 3.

\subsection{Comparison of analysis cases}

First, we compared the results of the six cases listed in Table 5: two lunar surface models and three damper models were used to find the best analysis model, in terms of accuracy and computation time. The velocity and acceleration of the body's center were chosen as the parameters for comparison, as shown in Fig. 9. When the lunar surface was assumed to be rigid, and the damper was also rigid, shock energy was not absorbed, as shown in Fig. 9 (a): the velocity changed suddenly, and the acceleration of the body's center reached its peak of $5,000 \mathrm{~m} / \mathrm{s}^{2}$. This means that the rigid surface-rigid damper model is not proper for landing simulations. When a viscous damper was used, the magnitude of acceleration was reduced, but was still large $\left(2,700 \mathrm{~m} / \mathrm{s}^{2}\right)$, as shown in Fig. 9 (b). However, when the $\mathrm{Al}$ honeycomb damper was used, the acceleration of the body's center was significantly reduced, to $100 \mathrm{~m} / \mathrm{s}^{2}$. This means that the $\mathrm{Al}$ honeycomb damper implementation explained in section 3.2 is proper.

When the lunar surface was modeled as an elastic surface, there were a lot of vibrations observed, as in Fig. 9 (d) (rigid damper), and Fig. 9 (e) (viscous damper). This may have resulted because the rigid damper and viscous damper were not able to properly absorb the impact energy. However, when the Al honeycomb damper was used for the same lunar surface condition, the elastic surface produced reasonable analysis results, as in Fig. 9 (f).

Table 6 shows the computation times of six cases, which were run on a computer $\left(\right.$ Intel $^{\circledR}$ core $^{\mathrm{TM}}$ i5-2500, 8192MB RAM). As shown, the computation times for the six cases are not very different, meaning there is no merit in using the rigid lunar surface-rigid damper model. So, the elastic surface and $\mathrm{Al}$ honeycomb damper model is preferred for the landing stability simulation.

Fig. 10 shows the force and stroke histories of the primary and secondary struts of the one-sixth lunar module, which was modeled with the elastic lunar surface and Al honeycomb damper model. When the lunar module impacted the lunar

Table 6. Comparison of calculation times

\begin{tabular}{cc}
\hline Cases & Calculation time (s) \\
\hline Rigid lunar surface-rigid damper & 58 \\
Rigid lunar surface-viscous damper & 56 \\
Rigid lunar surface-Al honeycomb damper & 88 \\
Elastic lunar surface-rigid damper & 60 \\
Elastic lunar surface-viscous damper & 58 \\
Elastic lunar surface-Al honeycomb damper & 119 \\
\hline
\end{tabular}

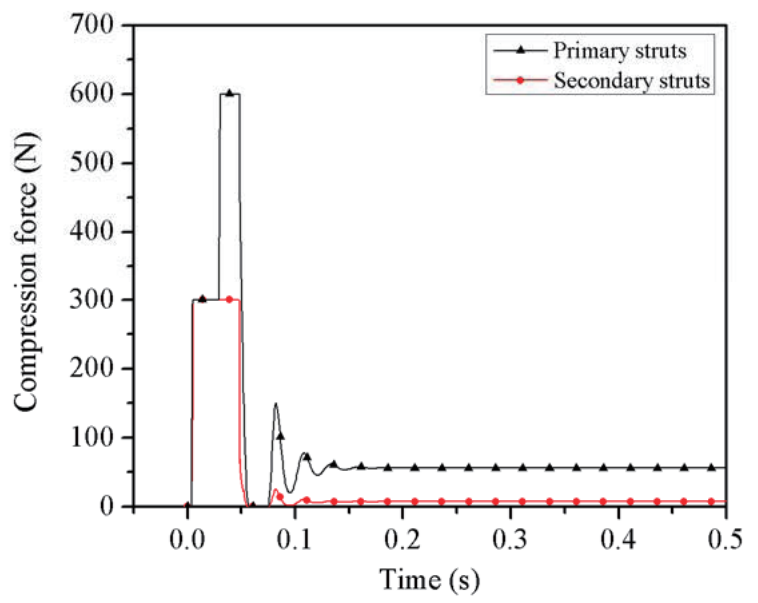

(a) Force histories of primary and secondary struts

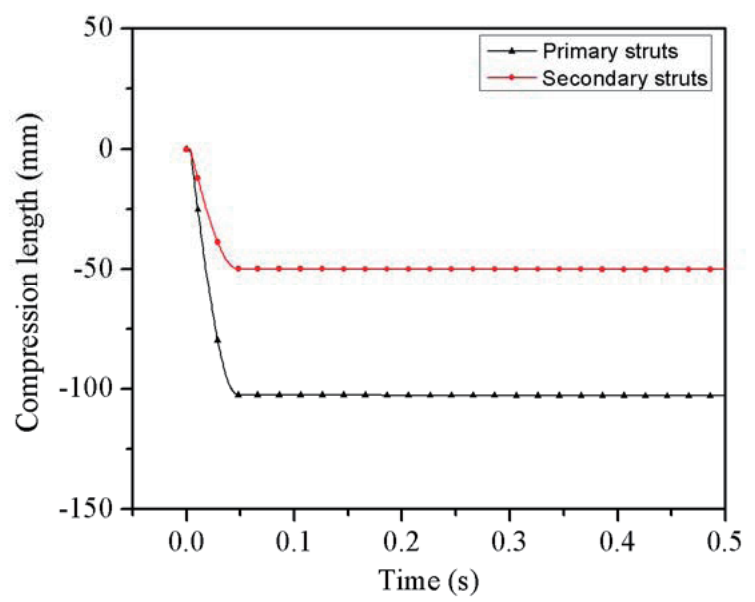

(b) Stroke histories of primary and secondary struts

Fig. 10. Force and stroke histories of primary and secondary struts of the one-sixth lunar module 
surface, the aluminum honeycomb shock absorbers of each primary strut started to compress. Due to the simultaneous impact and the symmetry of the model, the compression force and compression length for all the legs were the same. The force acting on the primary strut was so high, that the two stages of the primary struts were crushed. The crushing forces of the first and second stages were $300 \mathrm{~N}$ and $600 \mathrm{~N}$, respectively. However, the secondary struts suffered the crush of the first stage: they were subjected to a compression force of $300 \mathrm{~N}$ and a stroke of nearly $50 \mathrm{~mm}$, because most of the shock energy was absorbed in the primary struts. It can be concluded that most of the energy was absorbed by the aluminum honeycomb shock absorbers attached to the primary struts. Secondary struts were found to play minor roles in energy absorption.

\subsection{Landing response of the lunar module at an in- clined attitude}

When the lunar module touchdowns on the lunar surface, its attitude is unlikely to be perpendicular to the lunar surface. KARI's design criterion allows an inclined angle of 10 degrees. We investigated the landing responses of two typical modes: 2-2 landing mode and 1-2-1 landing mode, based on how the footpads make contact with the lunar surface; these are depicted in Fig. 11. In the 2-2 landing mode, two

Table 7. Comparison of compression length of primary struts

\begin{tabular}{cccc}
\hline $\begin{array}{c}\text { Primary } \\
\text { struts }\end{array}$ & $\begin{array}{c}\text { Level } \\
\text { landing }\end{array}$ & $\begin{array}{c}\mathbf{2 - 2} \\
\text { landing }\end{array}$ & $\begin{array}{c}\mathbf{1 - 2 - 1} \\
\text { landing }\end{array}$ \\
\hline $\begin{array}{c}\text { Leg\#1 } \\
(\mathrm{mm})\end{array}$ & 100.3 & 87.2 & 84.5 \\
$\begin{array}{c}\text { Leg\#2 } \\
(\mathrm{mm})\end{array}$ & 100.3 & 89 & 96.4 \\
$\begin{array}{c}\text { Leg\#3 } \\
(\mathrm{mm})\end{array}$ & 100.3 & 89.5 & 102.0 \\
$\begin{array}{c}\text { Leg\#4 } \\
(\mathrm{mm})\end{array}$ & 100.3 & 87.4 & 97.6 \\
\hline
\end{tabular}

\section{2-2 Landing mode}
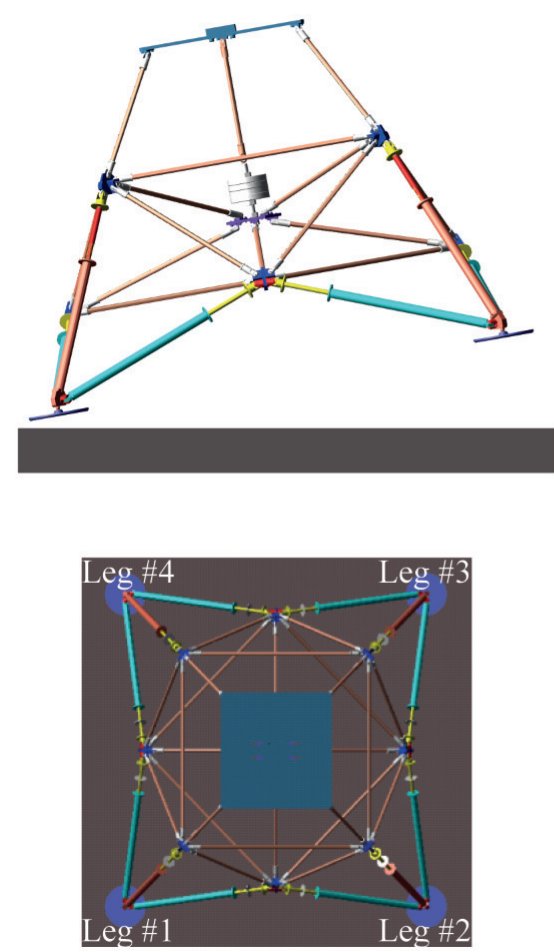

\section{1-2-1 Landing mode}
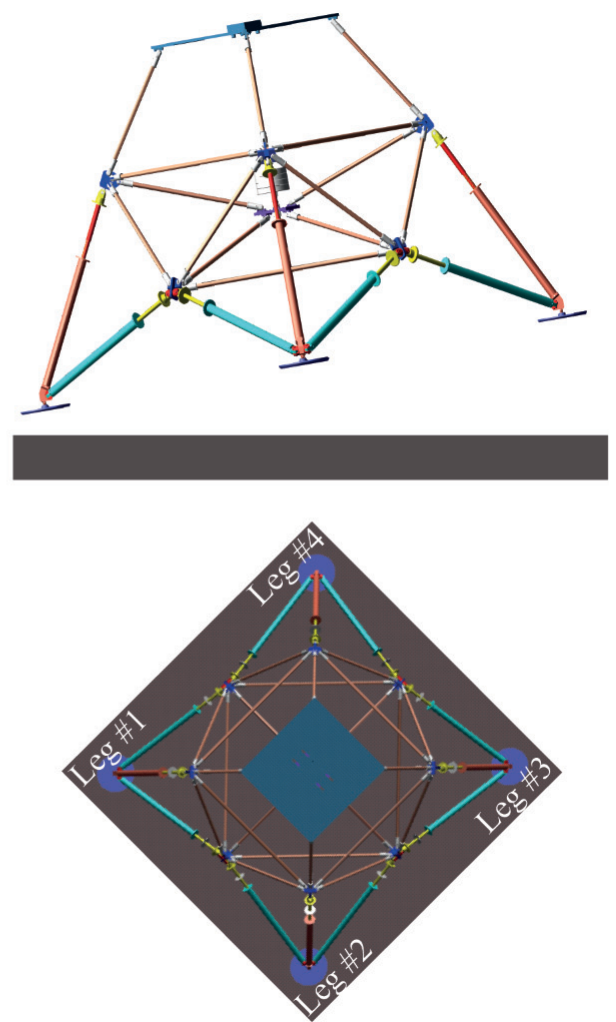

Fig. 11. Two typical landing modes: the 2-2 landing mode, and 1-2-1 landing mode 
front feet (leg\#1 and leg\#4) impact simultaneously, and then the two back feet (leg\#2 and leg\#3) impact simultaneously. In 1-2-1 landing mode, the front foot (leg\#1) makes initial contact with the surface, followed by a simultaneous impact of the two center feet (leg\#2 and leg\#4), and then the impact of the back foot (leg\#3).

\section{2-2 landing mode at inclined angle of 10 degrees}

The responses of the 2-2 landing mode on an elastic surface are investigated in this section. Fig. 12 shows the compression lengths of the primary struts, and the acceleration and velocity of the body's center. At the time the two front feet impact the surface, the impact energy is mainly absorbed in the primary struts of leg\#1 and leg\#4. Then, the other legs (leg\#2 and leg\#3) impact, and they too compress. Interestingly, leg \#2 and leg \#3 suffer compression between

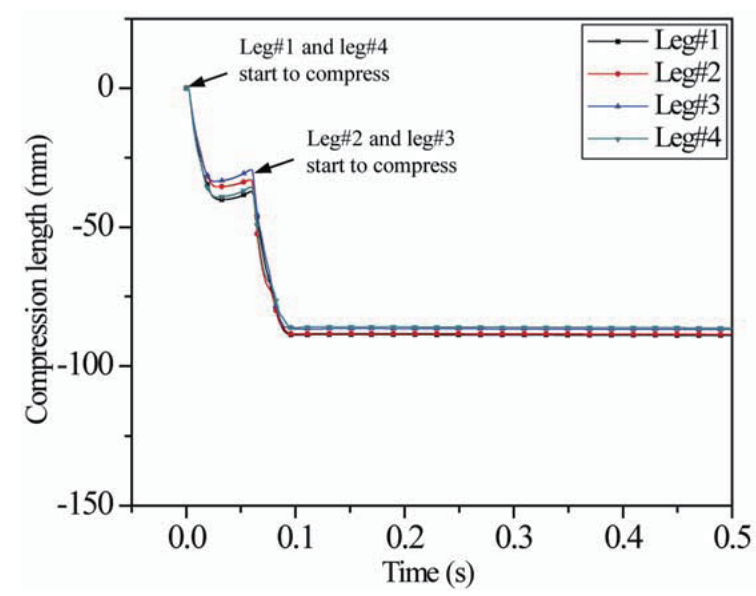

(a) Compression lengths of primary struts

Fig. 12. Responses of 2-2 landing mode, on an elastic surface

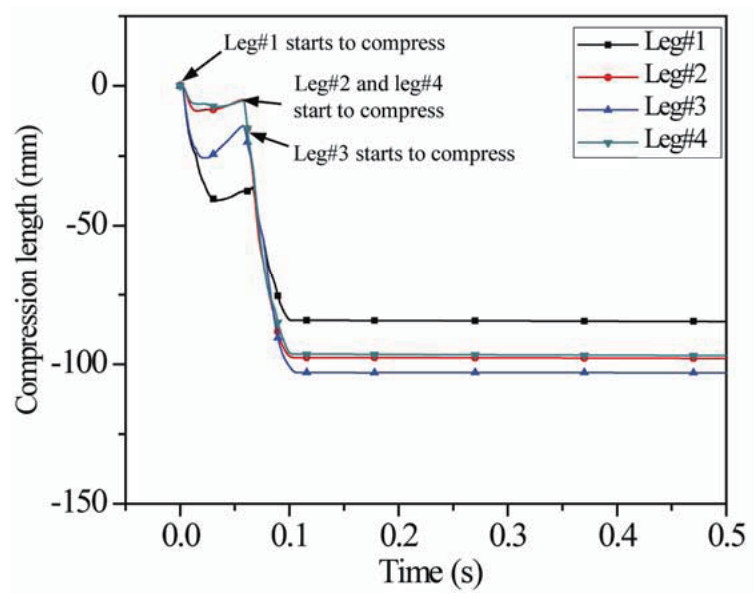

(a) Compression lengths of primary struts

Fig. 13. Responses of 1-2-1 landing mode, on an elastic surface the first two legs' impact and the second two legs' impact, because the lunar module is a truss structure, and the force at one leg can be transmitted to the structure. Also, leg\#2 and leg\#3 suffer from bigger compressive force than the other legs, due to the rotation of the body with respect to the body's center. Therefore, the front legs have larger compression lengths than the other legs. Since the compression length is around $90 \mathrm{~mm}$, the second stage in the primary strut is compressed, and the crush force is greater than $300 \mathrm{~N}$.

\section{1-2-1 landing mode at inclined attitude of 10 degrees}

Fig. 13 shows the compression lengths of the primary struts, and the acceleration and velocity of the body's center. Leg \# 1 contacts first, and so the other legs compress, as explained in the 2-2 landing mode case. After leg \#1 impacts, leg \#2 and leg \#4 impact, and then leg \#3 impacts. Similar

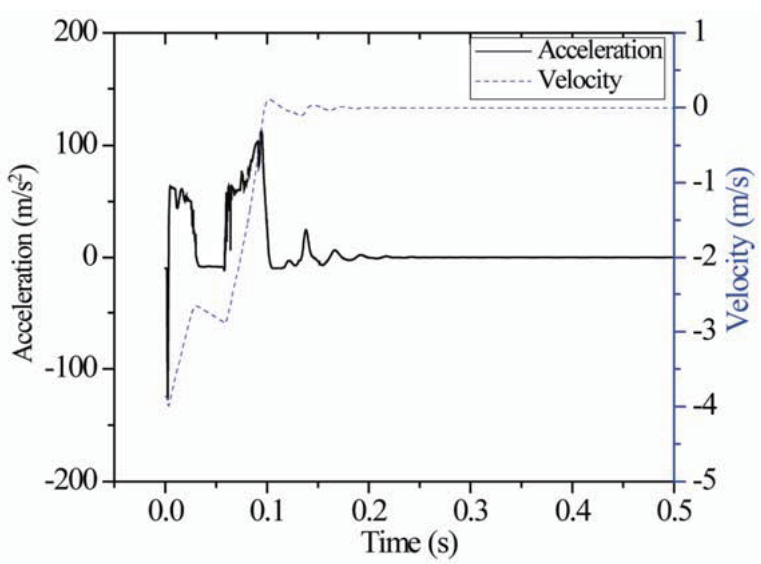

(b) Acceleration and velocity histories of body's center

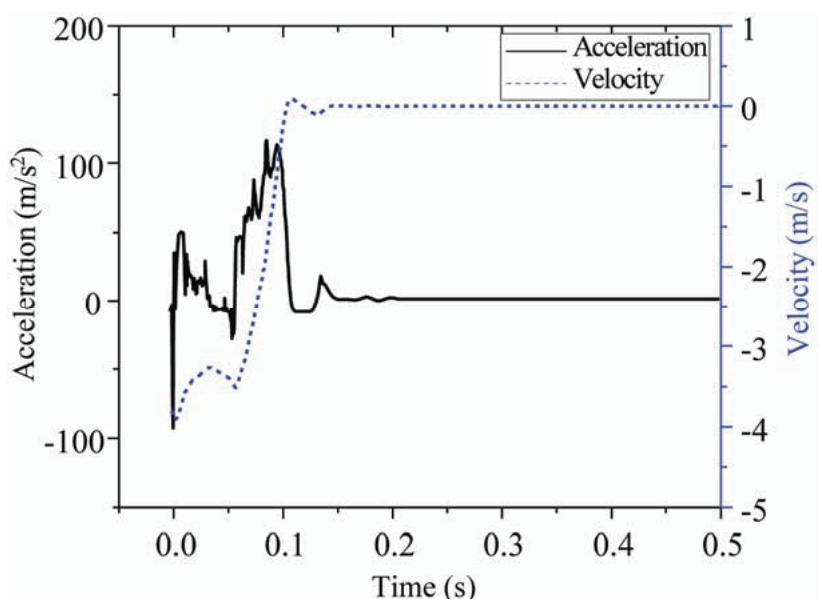

(b) Acceleration and velocity histories of body's center 
to the 2-2 landing mode, leg \#3 has the biggest compression length, which means the force acting on leg \#3 is the greatest.

Table 7 shows the compression lengths of the primary struts for level landing, 2-2 landing and 1-2-1 landing modes. The compression lengths for the 2-2 landing mode and the 1-2-1 landing modes are shorter than that for the level landing mode. This may be because the velocity perpendicular to the surface for the 2-2 landing and 1-2-1 landing modes is smaller, than the velocity for the level landing mode. There are extension periods of the honeycomb damper, after the first impact on the lunar surface, as shown in Figs. 12 (a) and 13 (a), although the honeycomb damper is always compressed in the level landing mode, as shown in Fig. 10 (b).

\subsection{Tip-over angle}

The landing stability on a tilted lunar surface was investigated. To calculate the tip-over angle of the lunar module, simulations were performed for increases of the slope of the lunar surface. The lunar surface was modeled as an elastic surface, and three kinds of damper models were considered: a rigid damper, viscous damper, and aluminum honeycomb damper. Also, two landing modes were compared, to determine which one provided the better landing stability. Tables 8 and 9 show the tip-over angles for the 2-2 landing mode and 1-2-1 landing mode, respectively.

The results show that the 1-2-1 landing mode is more stable than the 2-2 landing mode. The reason is that the moment arm between the body's center and back foot (leg \#3) in the 1-2-1 landing mode is larger, than the moment arm between the body's center and back foot (leg \#2 or leg \#3) in the 2-2 landing mode.

The results say that the rigid damper underestimates the tip-over angle, compared with the viscous and aluminum honeycomb dampers. It is concluded that the damping in the lunar module can increase the tip-over angle, since the damper can absorb the impact energy. The theoretical value

Table 8. Tip-over-angle for 2-2 landing mode, with different models of damper

\begin{tabular}{cc}
\hline Case & $\begin{array}{c}\text { Tip-over } \\
\text { angle }\left({ }^{\mathbf{0}}\right)\end{array}$ \\
\hline Rigid damper & 37 \\
$\begin{array}{c}\text { Model with viscous damper } \\
\text { Model with Al honeycomb shock } \\
\text { absorbers }\end{array}$ & 50 \\
\hline
\end{tabular}

Table 9. Tip-over angle for 1-2-1 landing mode, with different models of damper

\begin{tabular}{cc}
\hline Case & Tip-over angle ( $\left.{ }^{\mathbf{0}}\right)$ \\
\hline Rigid damper & 65 \\
$\begin{array}{c}\text { Model with viscous damper } \\
\text { Model with Al honeycomb shock } \\
\text { absorbers }\end{array}$ & 66 \\
\hline
\end{tabular}

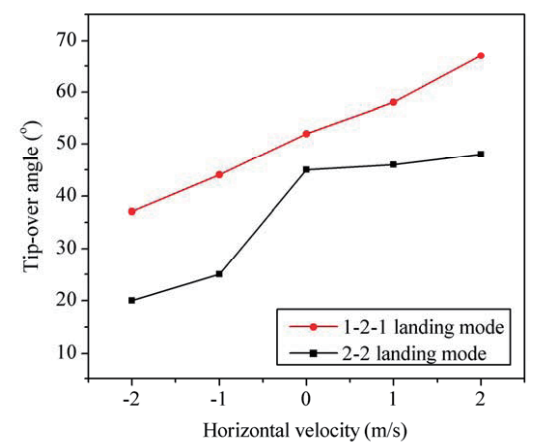

Fig. 14. Tip-over angles of lunar module for rigid damper, and rigid lunar surface

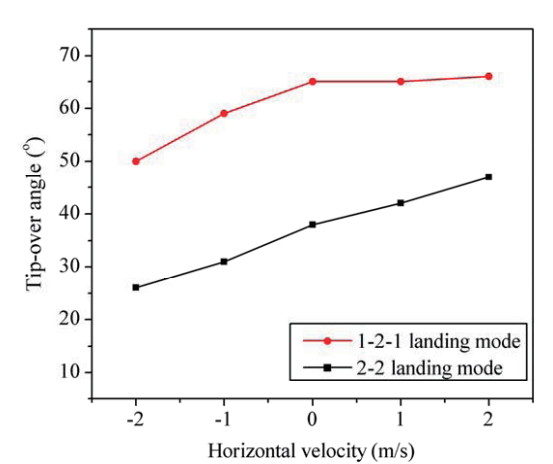

Fig. 15. Tip-over angles for rigid damper, and elastic surface 
for the tip-over angle calculated in section 2.2 was $40.3^{0}$, which is very close to that of the rigid damper case of the 2-2 landing mode $\left(37^{\circ}\right)$. This means that the rigid damper is assumed in the theory in section 2.2.

Horizontal velocity can affect the landing stability of the lunar module, as well as the vertical velocity. Since a rigid damper can give a conservative solution, the rigid damper model was preferred. Two lunar surface models were used, namely rigid and elastic surfaces. The positive horizontal velocity of the lunar module means the downward direction of the lunar slope. Figs. 14 and 15 show the tip-over angles of the lunar module for rigid and elastic surfaces, respectively. The tip-over angles for the 1-2-1 landing mode are greater than those of the 2-2 landing mode, which means that the 1-2-1 landing mode is more stable than the 2-2 landing mode.

\section{Conclusion}

In this paper, the touchdown dynamic model of a $1 / 6$ lunar module was built in ADAMS, and used in landing simulations, to develop a method for modeling an aluminum honeycomb damper using ADAMS, to compare the stability of the 2-2 landing and 1-2-1 landing modes, and to determine tip-over angles. Simulation results led to the following conclusions: (1) Aluminum honeycomb shock absorbers were modeled successfully in the multi-rigid-body dynamics software ADAMS/View, (2) Two typical landing modes were compared: the 2-2 landing mode and 1-2-1 landing mode. For rigid body impact, the 2-2 landing mode resulted in less stable landing than the 1-2-1 landing mode, (3) The model with the $\mathrm{Al}$ honeycomb shock absorbers was more stable than the model with the other dampers (rigid damper, and model with viscous damper).

The current simulation results will be confirmed with future landing tests, and the analysis technique developed in this work can be used for the detailed design of a lunar module structure.

\section{Acknowledgement}

This work was supported by the KARI-University Partnership Program. The authors are grateful for the financial support.

\section{References}

[1] Robert, W. H., and Leonard, H. W., "Dynamic model investigation of touchdown stability of lunar landing vehicles", NASA Technical Note D-4215, 1967.

[2] Sun, Y., Hu, Y., Liu, R. Q., and Den, Z. Q., “Touchdown dynamic modeling and simulation of lunar Lander", Systems and Control in Aeronautics and Astronautics (ISSCAA), 2010 3rd International Symposium on, 2010, pp.1320-1324.

[3] Jiang,W., Huang,W., and Shen, Z.,"Application of ADAMS user-written subroutine to simulation of softlanding dynamics", Spacecraft Recovery \& Remote Sensing 04, 2009, pp. 328-333.

[4] Lunar and Planetary Institute, Lunar Mission Summaries. Lunar Science and Exploration.

URL : http://www.lpi.usra.edu/lunar/missions/.

[5] ESA-NEXT Team, Next Lunar Lander with in-situ science and mobility: Lunar Environment Specification, European Space Agency, 2008.

[6] William, F. R., "Apollo Experience Report-Lunar Module Landing Gear Subsystem", NASA Technical Note D-6850, 1972.

[7] Johnson, K. L., Contact Mechanics, The Press Syndicate of The University of Cambridge, Cambridge, United Kingdom, 1987.

[8] Jiang, T., Hirotsugu , O., and Masahiko, H., "DynamicContact Stiffness at the Interface Between a Vibrating Rigid Sphere and a Semi-Infinite Viscoelastic Solid", IEEE Transactions on Ultrasonics, Ferroelectrics, and Frequency Control, Vol. 51, No. 14, 2004, pp. 1557-1563. 